\title{
Lyapunov-Type Inequalities for the Quasilinearq-Difference systems
}

\author{
Jiannan Song, YunfeiGao, ChengminHou* \\ (Department of Mathematics, Yanbian University, Yanji 133002, P. R. China)
}

\begin{abstract}
Using the Hölder inequality, we establish several Lyapunov-type inequalities for quasilinear $q$-difference equation and q-difference systems.
\end{abstract}

Keywords: Lyapunov-type inequalities, q-difference equation, q-difference systems, Hölderinequality

\section{Introduction}

The Lyapunov inequality and many of its generalizations have proved to be useful tools in oscillation theory, disconjugacy, eigenvalue problems, and numerous other applications for the theories of differential and difference equations. The well-known inequality of Lyapunov[1]states that a necessary condition for the boundary value problem $y^{\prime \prime}+q(t) y=0, y(a)=y(b)=0$, to have nontrivial solutions is that $\int_{a}^{b}|g(t)| d t>\frac{4}{b-a}$. There are several different proofs of this inequality since the original one by Lyapunov[1]. In the last few years independent works appeared generalizing Lyapunov's inequality for the $p$-Laplacian, by usingHölder, Jensen or Cauchy-Schwarz inequalities. For the case of a single equation, see for example [2-9]. For systems there are a few results.

In the paper, we consider boundary problem of the following quasilinear $q$-difference equation [10,11].

$$
\left\{\begin{array}{l}
-D_{q}^{+}\left(r(t)\left|D_{q}(u(t))\right|^{p-2}\left(D_{q}(u(t))\right)=f(t)|u(t)|^{p-2} u(t), t \in(0,1), 0<q<1 .\right. \\
u(0)=u(1)=0, u(t) \not \equiv 0, t \in(0,1) .
\end{array}\right.
$$

and boundary problem of quasilinear $q$-difference system

$$
\left\{\begin{array}{l}
-D_{q}^{+}\left(r_{1}(t)\left|D_{q}(u(t))\right|^{p_{1}-2}\left(D_{q}(u(t))\right)=f_{1}(t)|u(t)|^{\alpha_{1}-2} u(t)|v(t)|^{\alpha_{2}}, t \in(0,1), 0<q<1,\right. \\
-D_{q}^{+}\left(r_{2}(t)\left|D_{q}(v(t))\right|^{p_{2}-2}\left(D_{q}(v(t))\right)=f_{2}(t)|v(t)|^{\beta_{2}-2} v(t)|u(t)|^{\beta_{1}}, t \in(0,1), 0<q<1,(2)\right. \\
u(0)=u(1)=v(0)=v(1)=0, u(t) \not \equiv 0, v(t) \not \equiv 0, t \in(0,1) .
\end{array}\right.
$$

For the sake of convenience, we give the following hypothesis $\left(\mathrm{H}_{1}\right)$ and $\left(\mathrm{H}_{2}\right)$ for $(1)$ and hypothesis $\left(\mathrm{H}_{3}\right)$ for $(2)$ : $\left(\mathrm{H}_{1}\right) \quad r(t)$ and $f(t)$ are real-valued functions and $r(t)>0$ for all $t \in \mathbf{R} .1<p, p_{1}<\infty$, satisfy $\frac{1}{p_{1}}+\frac{1}{p}=1$.

( $\left.\mathrm{H}_{2}\right) 1<p_{1}, p_{2}<\infty, \alpha_{1}, \alpha_{2}, \beta_{1}, \beta_{2}>0$, satisfy $\frac{\alpha_{1}}{p_{1}}+\frac{\alpha_{2}}{p_{2}}=1$ and $\frac{\beta_{1}}{p_{1}}+\frac{\beta_{2}}{p_{2}}=1$.

We recall some concepts for $q$-difference operator. Throughout this paper, we assume $q \in(0,1)$.

The $q$-derivatives $D_{q} f$ and $D_{q}^{+} f$ of a function $f$ are given by

$$
\begin{gathered}
\left(D_{q} f\right)(x)=\frac{f(x)-f(q x)}{(1-q) x},\left(D_{q}^{+} f\right)(x)=\frac{f\left(q^{-1} x\right)-f(x)}{(1-q) x}, \text { if } x \neq 0 . \\
\left(D_{q} f\right)(0)=f^{\prime}(0) \text { and }\left(D_{q}^{+} f\right)(0)=q^{-1} f^{\prime}(0) \text { provided } f^{\prime}(0) \text { exists. }
\end{gathered}
$$

The $q$-integrals is defined by

$$
\int_{0}^{a} f(x) d_{q} x=(1-q) a \sum_{n=0}^{\infty} f\left(a q^{n}\right) q^{n}
$$




$$
\int_{a}^{b} f(x) d_{q} x=\int_{0}^{b} f(x) d_{q} x-\int_{0}^{a} f(x) d_{q} x .
$$

The $q$-analogue of Leibnitz rule is given by

$$
D_{q} f(x) g(x)=g(x) D_{q} f(x)+f(q x) D_{q} g(x) .
$$

An immediate consequence is the $q$-integration by parts rule (see[12]).

$$
\int_{a}^{b} g(x) D_{q} f(x) d_{q} x=f(b) g\left(q^{-1} b\right)-f(a) g\left(q^{-1} a\right)-\int_{a}^{b} f(x) D_{q}^{+} g(x) d_{q} x .
$$

\section{Lyapunov-Type Inequalities For $Q$-Difference Equation (1)}

In the section, we establish Lyapunov-type inequality for $q$-difference equation (1).

Denote

$$
\begin{aligned}
& \xi(t)=\left(\int_{0}^{t} r^{\frac{-p_{1}}{p}}(s) d_{q} s\right)^{\frac{p}{p_{1}}}=\left(\int_{0}^{t} r^{1-p_{1}}(s) d_{q} s\right)^{\frac{1}{p_{1}-1}}, \\
& \eta(t)=\left(\int_{t}^{1} r^{1-p_{1}}(s) d_{q} s\right)^{\frac{1}{p_{1}-1}} .
\end{aligned}
$$

Theorem2.1. Suppose that hypothesis $\left(\mathrm{H}_{1}\right)$ holds. If boundary value problem (1) has a solution. Then one has the following inequality:

$$
\int_{0}^{1} \frac{\xi(t) \eta(t)}{\xi(t)+\eta(t)} f^{+}(t) d_{q} t \geq 1,(3)
$$

where $f^{+}(t)=\max \{f(t), 0\}$.

Proof.By (1) and the $q$-integration by parts rule (*)

$$
\begin{aligned}
& -\int_{0}^{1} D_{q}^{+}\left(\left|r(t) \| D_{q} u(t)\right|^{p-2} D_{q} u(t)\right) u(t) d_{q} t \\
& =-\left.u(t)\left[r\left(q^{-1} t\right)\left|D_{q} u\left(q^{-1} t\right)\right|^{p-2} D_{q} u\left(q^{-1} t\right)\right]\right|_{0} ^{1}+\int_{0}^{1} r(t)\left|D_{q} u(t)\right|^{p-2} D_{q} u(t) D_{q} u(t) d_{q} t \\
& =\int_{0}^{1} r(t)\left|D_{q} u(t)\right|^{p} d_{q} t=\int_{0}^{1} f(t)|u(t)|^{p} d_{q} t .(4)
\end{aligned}
$$

By the boundary condition of (1), we have

$$
\begin{aligned}
& |u(t)|^{p}=\left|\int_{0}^{t} D_{q} u(s) d_{q} s\right|^{p} \leq\left(\int_{0}^{t}\left|D_{q} u(s)\right| d_{q} s\right)^{p}=\left(\int_{0}^{t} r^{\frac{-1}{p}}(s) r^{\frac{1}{p}}(s)\left|D_{q} u(s)\right| d_{q} s\right)^{p} \\
& \leq\left(\int_{0}^{t} r^{\frac{-p_{1}}{p}}(s) d_{q} s\right)^{\frac{p}{p_{1}}} \int_{0}^{t} r(s)\left|D_{q} u(s)\right|^{p} d_{q} s=\left(\int_{0}^{t} r^{1-p_{1}}(s) d_{q} s\right)^{\frac{1}{p_{1}-1}} \int_{0}^{t} r(s)\left|D_{q} u(s)\right|^{p} d_{q} s \\
& =\xi(t) \int_{0}^{t} r(s)\left|D_{q} u(s)\right|^{p} d_{q} s,
\end{aligned}
$$

and

$|u(t)|^{p}=\left|-\int_{t}^{1} D_{q} u(s) d_{q} s\right|^{p} \leq\left(\int_{t}^{1}\left|D_{q} u(s)\right| d_{q} s\right)^{p} \leq\left(\int_{t}^{1} r^{1-p_{1}}(s) d_{q} s\right)^{\frac{1}{p_{1}-1}} \int_{t}^{1} r(s)\left|D_{q} u(s)\right|^{p} d_{q} s$

$=\eta(t) \int_{t}^{1} r(s)\left|D_{q} u(s)\right|^{p} d_{q} s$.

Thus $|u(t)|^{p} \leq \frac{\xi(t) \eta(t)}{\xi(t)+\eta(t)} \int_{0}^{1} r(s)\left|D_{q} u(s)\right|^{p} d_{q} s$.

By (4), we have

$$
\begin{aligned}
& \int_{0}^{1} f^{+}(t)|u(t)|^{p} d_{q} t \\
& \leq \int_{0}^{1} \frac{\xi(t) \eta(t)}{\xi(t)+\eta(t)} f^{+}(t) d_{q} t \int_{0}^{1} r(t)\left|D_{q} u(t)\right|^{p} d_{q} t=\int_{0}^{1} \frac{\xi(t) \eta(t)}{\xi(t)+\eta(t)} f^{+}(t) d_{q} t \int_{0}^{1} f(t)|u(t)|^{p} d_{q} t
\end{aligned}
$$


$\leq \int_{0}^{1} \frac{\xi(t) \eta(t)}{\xi(t)+\eta(t)} f^{+}(t) d_{q} t \int_{0}^{1} f^{+}(t)|u(t)|^{p} d_{q} t .(5)$

Next, we prove that $\int_{0}^{1} f^{+}(t)|u(t)|^{p} d_{q} t>0$.(6)

If (6) is not true, then $\int_{0}^{1} f^{+}(t)|u(t)|^{p} d_{q} t=0$. (7)

From (4) and (7), we have

$$
0 \leq \int_{0}^{1} r(t)\left|D_{q} u(t)\right|^{p} d_{q} t=\int_{0}^{1} f(t)|u(t)|^{p} d_{q} t \leq \int_{0}^{1} f^{+}(t)|u(t)|^{p} d_{q} t=0 .
$$

It follows $D_{q} u\left(q^{n} t\right) \equiv 0, n=0,1,2, \ldots$, we obtain that $u(t) \equiv 0$, for $t \in(0,1)$ which contradicts the condition of (2). Therefore, from (5), we may see that (3) holds. The proof is completed.

Note that $\left(\frac{\xi+\eta}{2}\right)^{2} \geq \xi \eta$, one has following corollary 2.1 .

Corollary 2.1.Suppose that hypothesis $\left(\mathrm{H}_{1}\right)$ is satisfied. If $(1)$ has a solution $u(t)$. Then one has the following inequality:

$$
\int_{0}^{1}(\xi(t) \eta(t))^{\frac{1}{2}} f^{+}(t) d_{q} t \geq 2
$$

\section{Lyapunov-Type Inequalities For $Q$-Difference Equation (2)}

Denote

$$
\begin{aligned}
& \xi_{i}(t)=\left(\int_{0}^{t} r_{i}^{1-p_{i}}(s) d_{q} s\right)^{\frac{1}{p_{i}-1}}, i=1,2 .(8) \\
& \eta_{i}(t)=\left(\int_{t}^{1} r_{i}^{1-p_{i}}(s) d_{q} s\right)^{\frac{1}{p_{i}-1}}, i=1,2 .(9)
\end{aligned}
$$

Theorem3.1. Suppose that hypothesis $\left(\mathrm{H}_{2}\right)$ is satisfied. If system (2) has a solution $(u(t), v(t))$. Thenone has the following inequality:

$$
\begin{gathered}
\left(\int_{0}^{1} \frac{\xi_{1}(t) \eta_{1}(t)}{\xi_{1}(t)+\eta_{1}(t)} f_{1}^{+}(t) d_{q} t\right)^{\alpha_{1} \beta_{1} / p_{1}^{2}}\left(\int_{0}^{1} \frac{\xi_{1}(t) \eta_{1}(t)}{\xi_{1}(t)+\eta_{1}(t)} f_{2}^{+}(t) d_{q} t\right)^{\beta_{1} \alpha_{2} / p_{1} p_{2}} \\
\times\left(\int_{0}^{1} \frac{\xi_{2}(t) \eta_{2}(t)}{\xi_{2}(t)+\eta_{2}(t)} f_{1}^{+}(t) d_{q} t\right)^{\beta_{1} \alpha_{2} / p_{1} p_{2}}\left(\int_{0}^{1} \frac{\xi_{2}(t) \eta_{2}(t)}{\xi_{2}(t)+\eta_{2}(t)} f_{2}^{+}(t) d_{q} t\right)^{\alpha_{2} \beta_{2} / p_{2}^{2}} \geq 1 .
\end{gathered}
$$

where $f_{i}^{+}(t)=\max \left\{f_{i}(t), 0\right\}$, for $i=1,2$.

Proof. Similar to(4), we have

$$
\begin{aligned}
& \int_{0}^{1} r_{1}(t)\left|D_{q} u(t)\right|^{p_{1}} d_{q} t=\int_{0}^{1} f_{1}(t)|u(t)|^{\alpha_{1}}|v(t)|^{\alpha_{2}} d_{q} t,(10) \\
& \int_{0}^{1} r_{2}(t)\left|D_{q} v(t)\right|^{p_{2}} d_{q} t=\int_{0}^{1} f_{2}(t)|v(t)|^{\beta_{2}}|u(t)|^{\beta_{1}} d_{q} t,(11) \\
& |u(t)|^{p_{1}} \leq\left|\int_{0}^{t} D_{q} u(t) d_{q} t\right|^{p_{1}} \leq \xi_{1}(t) \int_{0}^{t} r_{1}(t)\left|D_{q} u(t)\right|^{p_{1}} d_{q} t \\
& |u(t)|^{p_{1}} \leq \eta_{1}(t) \int_{t}^{1} r_{1}(t)\left|D_{q} u(t)\right|^{p_{1}} d_{q} t, \\
& |u(t)|^{p_{1}} \leq \frac{\xi_{1}(t) \eta_{1}(t)}{\xi_{1}(t)+\eta_{1}(t)} \int_{0}^{1} r_{1}(t)\left|D_{q} u(t)\right|^{p_{1}} d_{q} t \cdot(12) \\
& \int_{0}^{1} f_{1}^{+}(t)|u(t)|^{p_{1}} d_{q} t \leq \mathrm{M}_{11} \int_{0}^{1} r_{1}(t)\left|D_{q} u(t)\right|^{p_{1}} d_{q} t=\mathrm{M}_{11} \int_{0}^{1} f_{1}(t)|u(t)|^{\alpha_{1}}|v(t)|^{\alpha_{2}} d_{q} t \\
& \leq \mathrm{M}_{11} \int_{0}^{1} f_{1}^{+}(t)|u(t)|^{\alpha_{1}}|v(t)|^{\alpha_{2}} d_{q} t
\end{aligned}
$$


$\leq \mathrm{M}_{11}\left(\int_{0}^{1} f_{1}^{+}|u(t)|^{p_{1}} d_{q} t\right)^{\frac{\alpha_{1}}{p_{1}}}\left(\int_{0}^{1} f_{1}^{+}(t)|v(t)|^{p_{2}} d_{q} t\right)^{\frac{\alpha_{2}}{p_{2}}} \cdot(13)$
$\int_{0}^{1} f_{2}^{+}(t)|u(t)|^{p_{1}} \leq \mathrm{M}_{12} \int_{0}^{1} f_{1}(t)|u(t)|^{\alpha_{1}}|v(t)|^{\alpha_{2}} d_{q} t \leq \mathrm{M}_{12} \int_{0}^{1} f_{1}^{+}(t)|u(t)|^{\alpha_{1}}|v(t)|^{\alpha_{2}} d_{q} t$

$\leq \mathrm{M}_{12}\left(\int_{0}^{1} f_{1}^{+}(t)|u(t)|^{p_{1}} d_{q} t\right)^{\frac{\alpha_{1}}{p_{1}}}\left(\int_{0}^{1} f_{1}^{+}(t)|v(t)|^{p_{2}} d_{q} t\right)^{\frac{\alpha_{2}}{p_{2}}}$.

where $\mathrm{M}_{11}=\int_{0}^{1} \frac{\xi_{1}(t) \eta_{1}(t)}{\xi_{1}(t)+\eta_{1}(t)} f_{1}^{+}(t) d_{q} t, \mathrm{M}_{12}=\int_{0}^{1} \frac{\xi_{1}(t) \eta_{1}(t)}{\xi_{1}(t)+\eta_{1}(t)} f_{2}^{+}(t) d_{q} t$.

Similar to the proof of(12),from(10)(11), we have

$$
|v(t)|^{p_{2}} \leq \frac{\xi_{2}(t) \eta_{2}(t)}{\xi_{2}(t)+\eta_{2}(t)} \int_{0}^{1} r_{2}(t)\left|D_{q} v(t)\right|^{p_{2}} d_{q} t .
$$

It follows from above form and the Hölder inequality that

$$
\begin{aligned}
& \int_{0}^{1} f_{1}^{+}(t)|v(t)|^{p_{2}} d_{q} t \leq \int_{0}^{1} \frac{\xi_{2}(t) \eta_{2}(t)}{\xi_{2}(t)+\eta_{2}(t)} f_{1}^{+}(t) d_{q} t \int_{0}^{1} r_{2}(t)\left|D_{q} v(t)\right|^{p_{2}} d_{q} t \\
& \leq M_{21} \int_{0}^{1} f_{2}^{+}(t)|u(t)|^{\beta_{1}}|v(t)|^{\beta_{2}} d_{q} t \\
& \leq M_{21}\left(\int_{0}^{1} f_{2}^{+}(t)|u(t)|^{p_{1}} d_{q} t\right)^{\frac{\beta_{1}}{p_{1}}}\left(\int_{0}^{1} f_{2}^{+}(t)|v(t)|^{p_{2}} d_{q} t\right)^{\frac{\beta_{2}}{p_{2}}} \cdot \\
& \int_{0}^{1} f_{2}^{+}(t)|v(t)|^{p_{2}} \leq M_{22}\left(\int_{0}^{1} f_{2}^{+}(t)|u(t)|^{p_{1}} d_{q} t\right)^{\frac{\beta_{1}}{p_{1}}}\left(\int_{0}^{1} f_{2}^{+}(t)|v(t)|^{p_{2}} d_{q} t\right)^{\frac{\beta_{2}}{p_{2}}} \cdot(16) \\
& \text { where } M_{21}=\int_{0}^{1} \frac{\xi_{2}(t) \eta_{2}(t)}{\xi_{2}(t)+\eta_{2}(t)} f_{1}^{+}(t) d_{q} t, M_{22}=\int_{0}^{1} \frac{\xi_{2}(t) \eta_{2}(t)}{\xi_{2}(t)+\eta_{2}(t)} f_{2}^{+}(t) d_{q} t .(17)
\end{aligned}
$$

Similar to (5), we have

$$
\begin{aligned}
& \int_{0}^{1} f_{1}^{+}(t)\left|u(t)^{p_{1}}\right| d_{q} t>0, \int_{0}^{1} f_{2}^{+}(t)\left|u(t)^{p_{1}}\right| d_{q} t>0, \\
& \int_{0}^{1} f_{1}^{+}(t)\left|v(t)^{p_{1}}\right| d_{q} t>0, \int_{0}^{1} f_{2}^{+}(t)\left|v(t)^{p_{2}}\right| d_{q} t>0 .
\end{aligned}
$$

From(14)-(16), (18),we have

$$
M_{11}^{\alpha_{1} \beta_{1}} / p_{1}^{2} M_{12}^{\beta_{1} \alpha_{2}} / p_{1} p_{2} M_{21}^{\beta_{1} \alpha_{2} / p_{1} p_{2}} M_{22}^{\alpha_{2} \beta_{2}} / p_{2}^{2} \geq 1 .
$$

Corollary 3.1. Suppose that hypothesis $\left(\mathrm{H}_{2}\right)$ are satisfied. If (2) has a solution $(u(t), v(t))$. Then one has the following inequality:

$$
\begin{aligned}
& \left(\int_{0}^{1} f_{1}^{+}\left(\xi_{1}(t) \eta_{1}(t)\right)^{\frac{1}{2}} d_{q} t\right)^{\beta_{1} \alpha_{2}} / p_{1} p_{2}\left(\int_{0}^{1} f_{2}^{+}\left(\xi_{2}(t) \eta_{2}(t)\right)^{\frac{1}{2}} d_{q} t\right)^{\beta_{1} \alpha_{2}} / p_{1} p_{2} \\
& \times\left(\int_{0}^{1} f_{1}^{+}\left(\xi_{1}(t) \eta_{1}(t)\right)^{\frac{1}{2}} d_{q} t\right)^{\beta_{1} \alpha_{2} / p_{1} p_{2}}\left(\int_{0}^{1} f_{2}^{+}\left(\xi_{2}(t) \eta_{2}(t)\right)^{\frac{1}{2}} d_{q} t\right)^{\beta_{1} \alpha_{2} / p_{1} p_{2}} \geq 2^{\left(p_{2} \beta_{1}+p_{1} \alpha_{2}\right) p_{1} p_{2}} .
\end{aligned}
$$

Next,we consider the quasilinear $q$-difference system involving the $\left(p_{1}, p_{2}, \ldots, p_{m}\right)$-Laplacian:

$$
\left\{\begin{array}{l}
-D_{q}^{+}\left(r_{1}(t)\left|D_{q} u_{1}(t)\right|^{p_{1}-2}\left(D_{q} u_{1}(t)\right)\right)=f_{1}(t)\left|u_{1}(t)\right|^{\alpha_{1}-2}\left|u_{2}(t)\right|^{\alpha_{2}} \ldots\left|u_{m}(t)\right|^{\alpha_{m}} u_{1}(t), \\
-D_{q}^{+}\left(r_{2}(t)\left|D_{q} u_{2}(t)\right|^{p_{2}-2}\left(D_{q} u_{2}(t)\right)\right)=f_{2}(t)\left|u_{1}(t)\right|^{\alpha_{1}}\left|u_{2}(t)\right|^{\alpha_{2}-2} \ldots\left|u_{m}(t)\right|^{\alpha_{m}} u_{2}(t), \\
\vdots \\
-D_{q}^{+}\left(r_{m}(t)\left|D_{q} u_{m}(t)\right|^{p_{m}-2}\left(D_{q} u_{m}(t)\right)\right)=f_{m}(t)\left|u_{1}(t)\right|^{\alpha_{1}}\left|u_{2}(t)\right|^{\alpha_{2}} \ldots\left|u_{m}(t)\right|^{\alpha_{m}-2} u_{m}(t) .
\end{array}\right.
$$

with boundary value conditions:

$$
u_{i}(0)=u_{i}(1)=0, u_{i}(t) \not \equiv 0, t \in(0,1), i=1,2, \cdots, m .(20)
$$

We give the following hypothesis $\left(\mathrm{H}_{3}\right)$. 
$\left(\mathrm{H}_{3}\right) \quad r_{i}(t)$ and $f_{i}(t)$ are real-valued functions and $r_{i}(t) \geq 0$ for $i=1,2, \cdots, m$.

Furthermore, $1<p_{i}<\infty$ and $\alpha_{i}>0$ satisfy $\sum_{i=1}^{m}\left(\alpha_{i} / p_{i}\right)=1$.

Denote

$$
\begin{aligned}
& \xi_{i}(t)=\left(\int_{0}^{t} r_{i}^{1-p_{i}}(s) d_{q} s\right)^{\frac{1}{p_{i}-1}} . \\
& \eta_{i}(t)=\left(\int_{t}^{1} r_{i}^{1-p_{i}}(s) d_{q} s\right)^{\frac{1}{p_{i}-1}} .
\end{aligned}
$$

Theorem3.2.Suppose that hypothesis $\left(\mathrm{H}_{3}\right)$ is satisfied. If system (19) has a solution $\left(u_{1}(t), u_{2}(t), \ldots ., u_{m}(t)\right)$ satisfying the boundary conditions(20), then one has the following inequality:

Proof.By $(19)\left(\mathrm{H}_{3}\right)$ and (20),we can get

$$
\prod_{i=1}^{m} \prod_{j=1}^{m}\left(\int_{0}^{1} \frac{\xi_{1}(\tau) \eta_{1}(\tau)}{\xi_{1}(\tau)+\eta_{1}(\tau)} f_{j}^{+}(\tau) d_{q} \tau\right)^{\alpha_{i} \alpha_{j} / p_{i} P_{j}} \geq 1
$$

$$
\int_{0}^{1} r_{i}(t)\left|D_{q} u_{i}(t)\right|^{p_{i}}=\int_{0}^{1} f_{i}(t) \prod_{k=1}^{m}\left|u_{k}(t)\right|^{\alpha_{k}} d_{q} t, i=1,2, \cdots, m .
$$

It follows from(21)and the Hölder inequality that

$$
\begin{aligned}
& \left|u_{i}(t)\right|^{p_{i}} \leq \xi_{i}(t) \int_{0}^{t} r_{i}(\tau)\left|D_{q} u_{i}(\tau)\right|^{p_{i}} d_{q} \tau . \\
& \left|u_{i}(t)\right|^{p_{i}} \leq \eta_{i}(t) \int_{t}^{1} r_{i}(\tau)\left|D_{q} u_{i}(\tau)\right|^{p_{i}} d_{q} \tau .
\end{aligned}
$$

Thus

$$
\begin{gathered}
\left|u_{i}(t)\right|^{p_{i}} \leq \frac{\xi_{i}(t) \eta_{i}(t)}{\xi_{i}(t)+\eta_{i}(t)} \int_{0}^{1} r_{i}(\tau)\left|D_{q} u_{i}(\tau)\right|^{p_{i}} d_{q} \tau, \\
\int_{0}^{1} f_{j}^{+}(t)\left|u_{i}(t)\right|^{p_{i}} d_{q} t \leq M_{i j} \prod_{k=1}^{m}\left(\int_{0}^{1} f_{i}^{+}(t)\left|u_{k}(t)\right|^{p_{k}}\right)^{\frac{\alpha_{k}}{p_{k}}},
\end{gathered}
$$

where $M_{i j}=\int_{0}^{1} \frac{\xi_{i}(t) \eta_{i}(t)}{\xi_{i}(t)+\eta_{i}(t)} f_{j}^{+}(t) d_{q} t, i, j=1,2, \ldots, m$. (23)

Similar to the proof of the (18), we have

$$
\int_{0}^{1} f_{i}^{+}(t)\left|u_{k}(t)\right|^{p_{k}} d_{q} t>0, i, k=1,2, \ldots, m .
$$

Therefore $\prod_{i=1}^{m} \prod_{j=1}^{m} M_{i j}^{\alpha_{i} \alpha_{j}} / p_{i} p_{j} \geq 1$. (24)

It follows from (23) and (24) that (23) holds.

Corollary 3.2. Suppose that hypothesis $\left(\mathrm{H}_{3}\right)$ aresatisfied. If system (19)has a solution $\left(u_{1}(t), u_{2}(t), \ldots, u_{m}(t)\right)$ satisfying(20),then onehas the following inequality:

$$
\prod_{i=1}^{m} \prod_{j=1}^{m}\left(\int_{0}^{1} f_{j}^{+}(\tau)\left(\left(\xi_{i}(t) \eta_{i}(t)\right)^{\frac{1}{2}}\right)^{\alpha_{i} \alpha_{j}} p_{i} p_{j} \geq 2 .\right.
$$

\section{References}

[1]. A.Lyapunov,Probleme General de la Stabilite duMouvement,in:Ann.Math.Studies,vol.17,Prin-ceton Univ.Press,1949,Reprinted from Ann. Fac.Sci.Toulouse,9(1907)207-474, Translation ofthe original paper published in Comm.Soc.Math.Kharkow. 1892.

[2]. R.P.Agarwal,C.-F.Lee,C.-C.Yeh,C.-H.Hong,Lyapunov and Writingerinequalities,Appl. Math.Lett.17(2004)847-853.

[3]. D.Cakmak,Lyapunov-type integral inequalities for certain higher order differential equations,Appl. Math.Comp 216(2010)368-373

[4]. A.Canada,J.A.Montero,S.Villegas,Lyapunov inequalities for partial differentialequations,J. Funct.Anal.237(2006)176-193.

[5]. M.K.Kwong, On Lyapunov inequality for disfocality,J.Math.Anal.Appl.83(1981)486-494. 
[6]. B.G.Pachpatte,Lyapunov type integral inequalities for certain differential equations, Georgian Math.J.4(1997)139-148.

[7]. J.P.Pinasco,Lower bounds for eigenvalues of the one-dimensional p-Laplacian,Abstr.Appl.Anal.2004(2004)147-153.

[8]. J.P.Pinasco,Comparison of eigenvalues for the $p$-Laplacian with integral inequalities,Appl. Math. Comput.182(2006)1399-1404.

[9]. X.Yang,K.Lo,Lyapunov-type inequality for a class of even-order differential equalitions,Appl. Math. Comput.215(2010)3884-3890

[10]. P.L.De Napoli,J.P.Pinasco,Estimates for eigebvalues of quasilinearellipticsystems,J.Differ-ential. Equations 227(2006)102-115

[11]. A.Tiryaki,M.Unal,D.Cakmak,Lyapunov-type inequalities for nonlinear systems,J.Math.Anal. Appl. 332(2007)497-511.

[12]. H.T.Koelink,T.H.Koornwinder, $q$-special functions, atutorial,in:M.Gerstenhaber,T.Stasheff

[13]. (Eds), Reformation Theory and Quantum Groups with Applications to Mathematical physics, in: Contemp. Math.,vol134,Amer.Math.soc.,providence.1992.DD.141-142. 